\title{
Generalization of Frequency Discrimination Learning Across Frequencies and Ears: Implications for Underlying Neural Mechanisms in Humans
}

\author{
Karine Delhommeau, ${ }^{1}$ Christophe Micheyl, ${ }^{2}$ and Roland Jouvent ${ }^{3}$ \\ ${ }^{1}$ Neuropsychology Department, Montreal Neurological Institute, 3801 University Street, Montreal, H3A 2B4, Canada \\ ${ }^{2}$ Research Laboratory of Electronics, Massachusetts Institute of Technology, Bldg 36-758, Cambridge, MA 02139-4307, USA \\ ${ }^{3}$ UMR CNRS 7593, Pavillon Clérambault La Salpetrière, Paris, France
}

Received: 29 September 2004; Accepted: 16 February 2005; Online publication: 18 April 2005

\begin{abstract}
Frequency discrimination thresholds (FDTs) at 750, 1500,3000 , and $6000 \mathrm{~Hz}$ were measured in 32 normal-hearing listeners before and after each listener practiced the task for $12 \mathrm{~h}$ at one of the above frequencies using a single ear. Marked improvements in thresholds taking place over several hours were observed during the frequency- and ear-specific training period. Comparisons between pre- and posttraining thresholds showed large improvements at the trained frequency, but also at other frequencies. The improvements were initially slightly-but significantlylarger at the trained frequency than at untrained frequencies. However, this trained-frequency advantage disappeared rapidly during the course of the twohour multifrequency posttraining session, suggesting rapid relearning or learning generalization across frequencies. In contrast, no significant ear specificity was found, not even at early stages of the posttraining session. These findings add to earlier results suggesting that, in humans, frequency discrimination learning is only weakly frequency-specific, and they reveal that a complete generalization across frequencies can occur rapidly with little retraining at the initially untrained frequencies. Implications regarding underlying mechanisms are discussed.
\end{abstract}

Correspondence to: Christophe Micheyl - Research Laboratory of Electronics - Massachusetts Institute of Technology - Bldg 36-758, Cambridge, MA 02139-4307, USA. Telephone: (617) 253-9840; fax: (617) 258-7003; email: cmicheyl@mit.edu
Keywords: audition, learning, frequency discrimination

\section{INTRODUCTION}

Performances in a variety of basic visual- and auditoryperception tasks can improve dramatically with practice. In the visual modality, for instance, spectacular perceptual-learning effects have been demonstrated in texture-, contrast-, orientation-, or movement-discrimination tasks (e.g., Ahissar and Hochstein 1996, 1997; Ball and Sekuler 1982; Fiorentini and Berardi 1980, 1981; Karni and Sagi 1991, 1993; Karni and Bertini 1997; Schoups and Orban 1996; Ahissar 2001; Gilbert 1994, 1996). In the auditory modality, considerable improvements in thresholds with practice have been shown in frequency- and duration-discrimination tasks (Ari-Even Roth et al. 2003; Campbell and Small 1963; Delhommeau et al. 2002; Demany 1985; Demany and Semal 2002; Grimault et al. 2002, 2003; Hawkey et al. 2004; Irvine et al. 2000; Wright and Fitzgerald 2005; Wright et al. 1997). The mechanisms responsible for these improvements remain unclear. They could involve a genuine change in sensory discrimination abilities (i.e., sensory learning), or they could reflect the subject's progressive adaptation to the cognitive demands of the task and test procedure (i.e., procedural or task learning) (Robinson and Summerfield 1996).

Auditory frequency discrimination is often taken as an example of sensory learning subtended by 
training-induced changes in plastic sensory maps inside the central nervous system. Specifically, training-related improvement in frequency discrimination thresholds (FDTs) in the Rhesus monkey were shown to be paralleled by enlargements of areas of the tonotopically organized primary auditory cortex (A1) that corresponded to the frequencies used during the training (Recanzone et al. 1993). These local changes in sensory maps effects are consistent with behavioral results obtained in the same animals, which indicate the improvement in FDTs to be limited to the narrow frequency range used during the training. A natural and important question is whether the same type of explanation also holds in humans. If frequency discrimination learning is mediated by similar neural mechanisms in humans and monkeys, then it should also be highly frequency-specific in humans. A review of existing psychoacoustical data on this question - which are considered in the discussion of the current article-suggests that the answer is still unclear. While some earlier studies conclude that frequency discrimination learning is strongly frequency-specific (Demany and Semal 2002), or at least somewhat frequency-specific (Irvine et al. 2000; Wright and Fitzgerald 2005; Wright et al. 1997), others conclude that it is not frequencyspecific (Demany 1985; Ari-Even Roth et al. 2003; Grimault et al. 2003), at least within the frequency range tested. The reasons for such different outcomes across studies are unclear. They are likely to relate, at least in part, to the use of different experimental designs.

The primary aim of the present study was to test for differences in how FDTs improve with training at different frequencies. While this has not been looked at in detail in previous studies, differences in the time course of learning at different frequencies might help to clarify discrepancies in outcomes among earlier studies. For instance, Demany and Semal's (2002) finding that FDTs at 1200 and $6500 \mathrm{~Hz}$ can still improve markedly with practice after FDTs at 3000 $\mathrm{Hz}$ have ceased to do so, which these authors interpreted as evidence for frequency-specific learning, might be explained differently if, for some reason, FDT improvements were found to take longer at the latter than at the former two frequencies. There are various reasons for which training-induced improvements in FDTs should not necessarily have the same time course at all frequencies. For instance, certain frequencies may be more important than others for communication in natural settings, and discrimination abilities at these frequencies may already be enhanced prior to the psychoacoustical training.

Another main objective of the present study was to provide further data on the influence of long-term training at a single frequency on FDTs at other fre- quencies, distributed over a relatively wide rangebetween 750 and $6000 \mathrm{~Hz}$. In particular, we looked at how FDTs at the trained and other frequencies varied during the course of a relatively long (two-hour) posttraining session. Specifically, we were interested in whether the learning, although initially frequencyspecific, would rapidly generalize across frequency as listeners were again exposed to multiple test frequencies. If so, this might also contribute to clarify some of the discrepancies between earlier studies, some of which used very brief posttraining sessions (e.g., Irvine et al. 2000).

Finally, another objective assigned to the present study was to provide further data on how frequency discrimination learning transfers across the right and left ears. While the above-cited study by Demany and Semal (2002) and a recent study by Ari-Even Roth et al. (2004) showed no significant ear-specificity in frequency discrimination learning, a former study by Delhommeau et al. (2002) showed a slight, but significant, ear-specific component. Thus, half of the listeners in the present study were trained with stimuli presented to the right ear and the other half was tested with stimuli presented to the left ear.

\section{MATERIAL AND METHODS}

\section{Subjects}

Thirty-two adult (mean age $=21$ years, $\mathrm{SD}=1.42$ ) male subjects with normal-hearing (pure-tone hearing thresholds $\leq 20 \mathrm{~dB}$ HL at octave frequencies between 500 and $8000 \mathrm{~Hz}$ ) took part in the study. They had no prior experience with psychoacoustical tests and no particular musical training. They were paid an hourly wage for their services. Testing took place in a quiet room. In accordance with the requirements of the ethics committee from which approval for this study was received, written informed consent was obtained from each subject prior to testing.

\section{Stimuli and procedure}

All subjects took part in an initial test session, which began with the measurement of absolute hearing thresholds at four different test frequencies (750, 1500,3000 , and $6000 \mathrm{~Hz}$ ) using a two-interval, twoalternative, forced choice (2I-2AFC) procedure with a two down, one up adaptive level variation rule tracking $70.7 \%$ correct points on the psychometric function. The measured absolute thresholds were used to adjust the level of the tones in the frequency discrimination test: the tones were presented at $50 \mathrm{~dB}$ SL, i.e., $50 \mathrm{~dB}$ above the absolute threshold 
measured at the corresponding frequency. FDTs were measured using a three-interval, two-alternative, forced choice (3I-2AFC) procedure, which tracked $70.7 \%$ correct points on the psychometric function. Each trial involved the presentation of three successive tones of $200 \mathrm{~ms}$ each (including 20-ms cosine ramps) separated by $200-\mathrm{ms}$ silent intervals. The first tone had a frequency, $F$, which was equal to one of the above frequencies $(750,1500,3000$, or $6000 \mathrm{~Hz}$ ) and which remained constant throughout a block of trials or "run." This reference tone was followed by two tones, one of the same frequency, the other of frequency $F+\Delta F \mathrm{~Hz}$, where $\Delta F$ represents a variable frequency increment. At the beginning of a block of trials, $\Delta F$ was equal to $20 \%$ of the standard frequency. The order of presentation of the latter two tones was chosen randomly, the two possible orders of presentation being equally likely. The subject's task was to indicate which of the last two intervals contained the tone with a different frequency, i.e., they had to "tell the odd one out." Responses were given using keys " 2 " and " 3 " on a PC's numerical keypad. After two consecutive correct responses, the size of the frequency difference, $\Delta F$, was divided by 2 (until the fourth reversal, or $\sqrt{ } 2$ after the fourth reversal); $\Delta F$ was multiplied by the same factor after any incorrect response. The procedure stopped after a total of 12 reversals, and the FDT was computed as the geometric mean of $\Delta \mathrm{F}$ at the last four reversals. A run typically involved around 50 trials.

During the initial test session, FDTs were measured at each of the four different test frequencies, in each ear-the stimuli being always presented monaurally. The eight different frequency $\times$ ear combinations yielded eight testing and training conditions. Three threshold measurements (runs) were performed in each condition, resulting in a total of 24 threshold measurements in total on this "pretraining" session. The different conditions were tested in completely random order, i.e., the order was generally different across subjects, and not the same between the pre- and posttraining sessions.

After this initial test session, the subjects took part in six successive training sessions proper. These took place on different days, with three sessions per week. During each training session, the subjects performed 15 runs of the FDT measurement procedure, leading to a total of 90 runs over the whole two-week training period. For a given subject, the standard frequency $F$ was maintained constant throughout these 90 runs, and the tones were always presented in the same ear. Eight subjects were trained using a standard frequency $F$ of $750 \mathrm{~Hz}$. Four of these subjects were trained with the stimuli presented in the right ear, the other half being trained with stimuli in the left ear. Three similar subject groups were formed for the other three frequencies $(1500,3000$, and $6000 \mathrm{~Hz})$. Thus, the study involved 32 subjects overall.

After completing the six training sessions, the subjects took part in a posttraining session, which took place on a different day than the last training session. During this posttraining session, FDTs were measured three times at each of the four test frequencies, in each ear-just like on the pretraining session.

\section{Apparatus}

The stimuli were generated in the time domain using a Pentium computer. A 44.1-kHz sampling rate and a 16-bit dynamic range were used. The digital waveforms were converted to analog signals and played out using a Sound Blaster Pro audio card. The signals were delivered to the subject's right or left ear via Sony MDR-CD470 headphones.

\section{RESULTS}

Pre- and posttraining thresholds

The FDTs measured at the different test frequencies on the pre- and posttraining sessions are shown in Figure 1. All FDTs in this article are expressed as $\Delta F / F$ in percent, plotted on a logarithmic scale, and were log-transformed before statistical analyses. While some variability across groups is apparent in both the pre- and the posttraining data, overall, no significant difference in FDTs across groups was observed on these two sessions $\left(F_{3,28}=0.078, p=0.97\right.$ for the pretraining session; $F_{3,28}=0.13, p=0.94$ for the posttraining session). There was no significant difference in FDTs between right and left ears either $\left(F_{1,28}=0.95, p=0.34\right.$ for the pretraining session; $F_{1,28}=1.25, p=0.27$ for the posttraining session). On the other hand, there was a highly significant effect of frequency on both sessions $\left(F_{3,84}=53.63, p<\right.$ 0.00001 for the pretraining session; $F_{3,84}=74.01, p<$ 0.00001 for the posttraining session): FDTs were smallest at $1500 \mathrm{~Hz}$ and largest at $6000 \mathrm{~Hz}$. There was also a highly significant main effect of session $\left(F_{1,28}=\right.$ $158.09, p<0.00001)$ : FDTs were generally smaller on the post- than on the pretraining session, confirming the occurrence of perceptual learning. No significant interaction was observed between the session and training-frequency factors $\left(F_{3,28}=0.65, p=0.59\right)$, or between the session and test-ear factors $\left(F_{1,28}=\right.$ $0.0014, p=0.97)$. There was no significant interaction between the session and test-frequency factors either $\left(F_{3,84}=1.61, p=0.19\right)$. In other words, similar global improvements were obtained at all test frequencies and in both ears, irrespective of which frequency and ear were used during the training. However, as ex- 


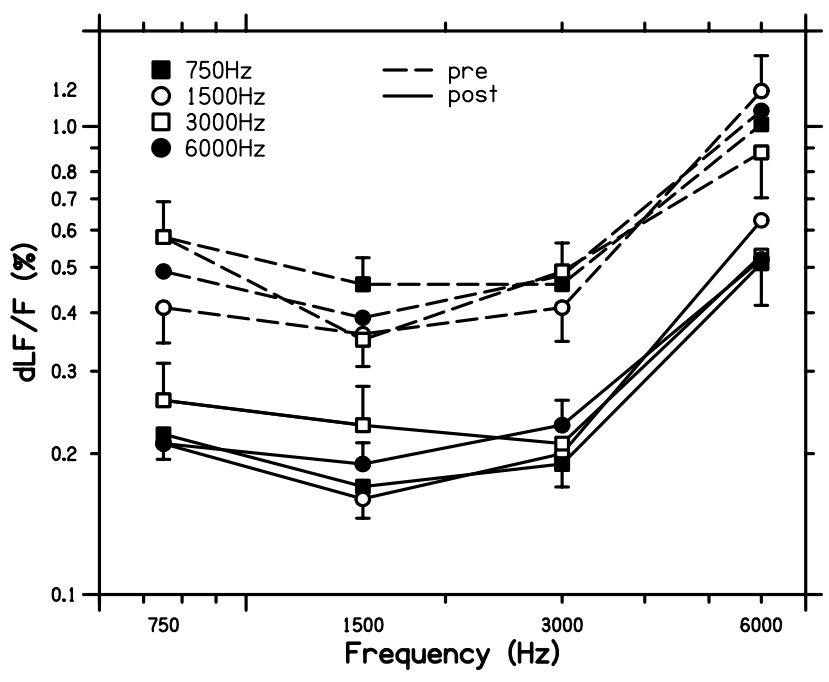

FIG. 1. Pre- and posttraining FDTs at the different test frequencies and in the different training groups. The different training groups/ frequencies are identified by different symbols. The test frequencies are indicated on the abscissa. Pretraining data are connected by dashed lines; posttraining data, by solid lines. Consistent with the use of geometric averages and with the fact that statistical analyses were performed on the log-transformed FDTs, the error bars in this and following figures represent geometric standard errors, which were computed as the exponential of the standard errors of the logtransformed FDTs (in percent). The resulting values are factors rather than additive quantities, so that they yield symmetric plus and minus error bars when plotted on a log scale.

plained below, this outcome was qualified by the results of more detailed analyses.

\section{Learning curves}

Figure 2 shows how the FDTs improved during the six frequency-specific training sessions that followed the multifrequency pretraining session. A general linear model (GLM) analysis was performed on these learning data, using the trained frequency and the trained ear as categorical factors, the run number as linear predictor, and the log-transformed thresholds as dependent variable. The results showed significant differences in learning slopes between frequencies $\left(F_{3,2864}=3.70, p=0.01\right)$. Planned comparisons (Table 1) revealed significantly shallower slopes at $1500 \mathrm{~Hz}$ than at the other frequencies. In fact, and by contrast with the other training frequencies, in five out of eight subjects trained at $1500 \mathrm{~Hz}$, the slopes of the lines fitted through the learning data were found to be nonsignificantly different from zero, suggesting no significant improvement during the training period in roughly half of the subjects at that frequency. Because $1500 \mathrm{~Hz}$ was also the frequency at which FDTs were initially the smallest, we decided to test whether the slopes of the learning functions were generally related to the pretraining thresholds. Indeed, a significant correlation was observed between pretraining FDTs and learning-function slopes (Pearson's $r=-0.46, p=0.009$ ).

To determine whether the finding of shallower learning curves in the $1500-\mathrm{Hz}$ group could be explained by something unusual about this group, other than the particular training frequency used, we tested whether listeners in this group had unusually low thresholds (at all frequencies) prior to training. An ANOVA contrasting pretraining FDTs in the $1500-\mathrm{Hz}$ training group with FDTs in the other training groups, for all frequencies, showed no significant difference across groups $\left(F_{1,28}=0.15, p=\right.$ $0.70)$. We also tested, more specifically, whether pretraining FDTs measured at the sole $1500-\mathrm{Hz}$ frequency were unusually smaller in the $1500-\mathrm{Hz}$ training group than in the other groups. Again, the answer was negative $\left(F_{1,28}=0.13, p=0.72\right)$. Therefore, it appears that there was nothing unusual about the listeners making up the $1500-\mathrm{Hz}$ training group. The finding of unusually shallow learning curves in this group seems to be specific to the training frequency used in this group $(1500 \mathrm{~Hz})$, rather than to the group itself.

The apparent discrepancy between our finding of similar pre-to-post improvements across all frequen-

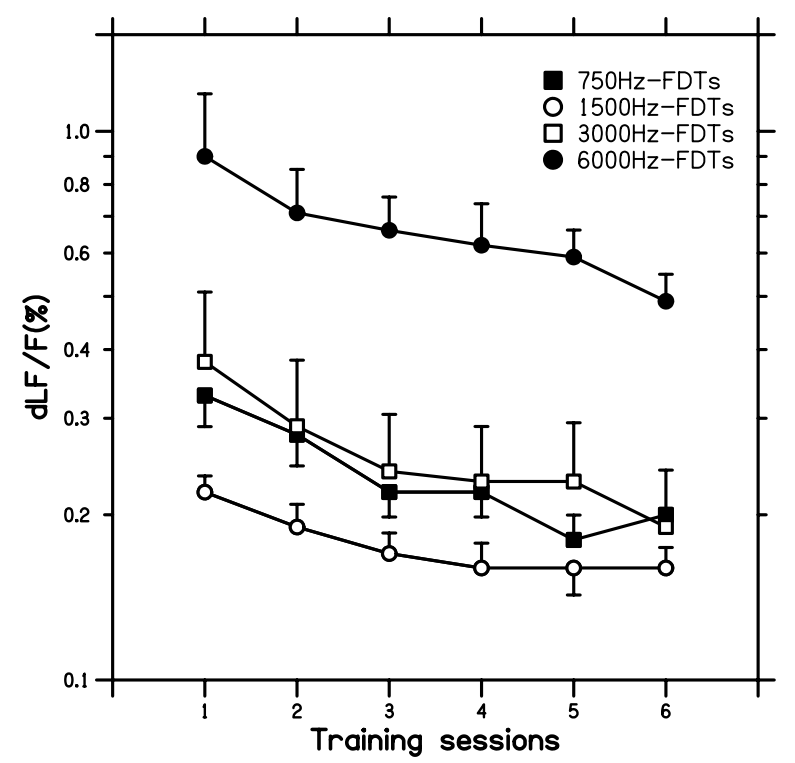

FIG. 2. FDTs measured on consecutive training sessions in each frequency-training group. The symbols identifying the different training groups/frequencies are the same as in the previous figure. Each of the connected data points is the (geometric) mean of 15 thresholds measured on each session in each of eight listeners (a total of 120 threshold estimates per point). The (geometric) mean FDTs at the training frequency corresponding to each training group are also shown here as unconnected data points on the left and right for the pre- and posttraining sessions. The error bars show geometric standard errors. 


\section{TABLE 1}

Comparisons between the slopes of the regression lines fitted through the frequency-specific training data for the different training-frequency groups

\begin{tabular}{lcc}
\hline Training frequencies $(\mathrm{Hz})$ & $F_{1,1436}$ & $P$ \\
\hline 750 vs. 1500 & 9.26 & $<0.005$ \\
750 vs. 3000 & 0.01 & 0.91 \\
750 vs. 6000 & 0.80 & 0.37 \\
1500 vs. 3000 & 7.42 & $<0.01$ \\
1500 vs. 6000 & 4.49 & $<0.05$ \\
3000 vs. 6000 & 0.78 & 0.38 \\
\hline
\end{tabular}

cies in the previous section and the current finding of shallower learning slopes in the $1500-\mathrm{Hz}$ training group may be explained by noticing in Figure 2 that while in all the other training groups thresholds increased visibly between the last frequency-specific training session and the posttraining session, in the $1500-\mathrm{Hz}$ training group, they did not. Because of this effect, the pre-to-post difference in thresholds was as large in the $1500-\mathrm{Hz}$ training group than in the other groups, despite the fact that thresholds improved significantly less during the training period itself. We cannot offer an explanation for why FDTs at the training frequency did not increase between the last frequency-specific training session and the posttraining session in the $1500-\mathrm{Hz}$ group. We expected changes in the test frequency across blocks of trials to have some detrimental influence on FDTs at all frequencies and in all listeners. The present results indicate that this is not always the case.

Finally, it is worth mentioning that no significant overall difference was found between the groups trained in the right and left ears $\left(F_{1,2864}=0.54, p=\right.$ $0.46)$, and that no significant interaction was observed either between the frequency and training-ear factors $\left(F_{3,286}=0.54, p=0.46\right)$, indicating that the FDTs measured in the right and left ears during the frequency-specific training sessions were of similar size and improved similarly.

\section{Detailed comparisons of pre- and} posttraining thresholds

The results described in "Pre- and posttraining thresholds" indicate that, on the whole, FDTs did not improve more at the trained frequency than at untrained frequencies. The ratio of pre-to-post training FDTs at trained frequencies was only slightly and nonsignificantly larger than the ratio at untrained frequencies (2.30 vs. 2.01 , respectively; $F_{1,120}=1.47$, $p=0.22)$. Thus, at first sight, there was no evidence for significantly larger improvements at the trained frequency than at untrained ones. However, this ap- parently complete lack of frequency-specific learning was qualified by a more in-depth analysis of the data. Specifically, we observed that the FDTs improved across the three runs performed within the course of the pre- and posttraining sessions. This is illustrated in Figure 3, and it was confirmed by statistical analyses $\left(F_{2,240}=7.06, p=0.001\right.$ for the pretraining session; $F_{2,240}=3.91, p=0.021$ for the posttraining session). Importantly, on the posttraining session, a larger improvement was observed between the first two runs for untrained frequencies than for the trained frequency. This was related to the fact that, on this posttraining session, FDTs at untrained frequencies were initially larger than FDTs at the trained frequency. However, by the second run of that session, the FDTs at untrained frequencies had already decreased enough to equal the FDTs measured at the trained frequency. When contrasting the FDTs measured on the last run of the pretraining session with FDTs measured on the first run of the posttraining session, we found a significant session $\times$ condition interaction $\left(F_{1,120}=4.47, p=0.036\right)$; however, when contrasting the third run of the pretraining session and the third run of the posttraining session, the interaction was no longer present $\left(F_{1,120}=2.56, p=0.11\right)$.

A similar detailed analysis, when applied to the training-ear factor, showed that the lack of difference in threshold improvement between the right and left ears, which was found initially when including all three runs of the pre- and posttraining sessions, remained true even when the runs were analyzed sepa-

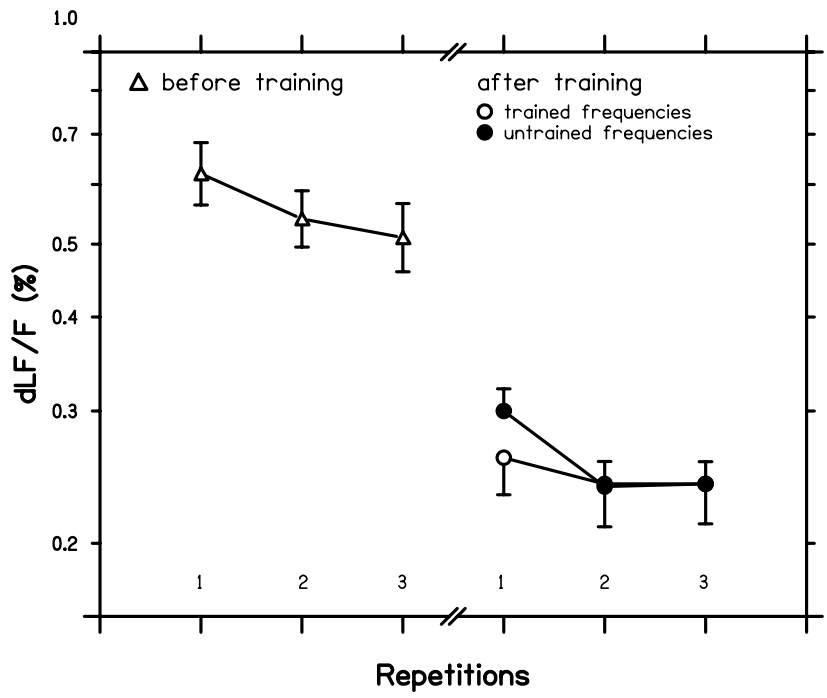

FIG. 3. FDTs measured at trained and untrained frequencies on each of the three runs of the pre- and posttraining sessions. Pretraining data are represented by upward-pointing triangles; posttraining data, by circles. Open circles correspond to posttraining FDTs at the frequency used during the frequency-specific training; filled circles, to posttraining FDTs obtained at the other frequencies. The error bars show geometric standard errors. 
rately. This confirms the general lack of ear effect in this study.

\section{DISCUSSION}

\section{Differences in learning rate across frequencies}

The first objective assigned to this study was to test for differences in the rate or extent of improvements in FDTs across frequencies. The results showed some evidence for such differences. Specifically, the slope of the line fitted through the training data of the group trained at $1500 \mathrm{~Hz}$ was significantly smaller than the slopes measured in other training groups, using other training frequencies. Because the preand posttraining thresholds measured in the $1500-\mathrm{Hz}$ training group were not significantly different from those measured in the other training groups, we suggest that the present finding of shallower learning functions in this group reflects a specificity of the $1500-\mathrm{Hz}$ frequency, rather than a particularity of the $1500-\mathrm{Hz}$ training group. However, we acknowledge that further data are needed before a firm conclusion can be reached on this point. Currently, there are not enough data in the literature to support the present results suggesting that the time course of frequency discrimination learning is different at $1500 \mathrm{~Hz}$ than at other frequencies. Furthermore, we can only speculate as to why less marked improvements in FDTs during the frequency-specific training period were observed at $1500 \mathrm{~Hz}$ than at the other frequencies tested here. Although we pointed out above that $1500 \mathrm{~Hz}$ happened to be the frequency at which the FDTs measured in this study were the smallest, the validity of this assertion depends on the particular choice of units; had FDTs been expressed in $\mathrm{Hz}$ rather than as percents of the reference frequency, this assertion would not have been true. Even if a significant correlation was found between the pretraining FDTs and the slope of the learning functions, the hypothesis that initial thresholds can determine subsequent improvements needs further scrutiny. Finally, although we pointed out in the Introduction that differences in the time course of learning across frequency might provide an alternative explanation for Demany and Semal's (2002) findings of additional improvements at 1200 and $6500 \mathrm{~Hz}$ after protracted training at $3000 \mathrm{~Hz}$, the present results do not conclusively support the hypothesis that FDTs cease to improve earlier at $3000 \mathrm{~Hz}$ than at other frequencies. On the other hand, the present finding of shallower learning functions at $1500 \mathrm{~Hz}$ suggests that the time course of frequency discrimination learning is not necessarily the same at all frequencies, and that this possibility should be seriously taken into account when interpreting the results of experiments involving an initial training phase at one frequency followed by retraining at other frequencies.

\section{Transfer of learning across frequencies}

The second main result obtained in the present study relates to the question of whether and how frequency discrimination learning generalizes across frequencies. While global comparisons between the pre- and posttraining results initially suggested that the learning had generalized completely across frequencies, a more in-depth analysis of the results revealed a different picture. Specifically, it was found that when only the earliest runs of the posttraining session were included in the comparison between the pre- and posttraining sessions, slightly but significantly larger improvements were observed at the trained frequency than at untrained frequencies. This significant frequency-specific component of the learning, however, disappeared rapidly within the course of the posttraining session: by the end of that session, the improvement was no longer significantly larger at the trained frequency than at untrained ones. This result cannot be explained by increasing fatigue during the posttraining session, because thresholds did not become substantially worse across successive runs during that session; on the contrary, as illustrated above, they improved. Instead, it appears that the reason for the disappearance of the frequency-specific component of the learning on the posttraining session was that, during that session, thresholds at the previously trained frequencies were lower, and improved less, than thresholds at previously untrained frequencies.

One interpretation of this pattern of results is that frequency discrimination learning, while partly frequency-specific, can nevertheless generalize rapidly across frequencies when listeners are again exposed to tones at multiple frequencies intermingled within the same session. Earlier experimental results in the visual modality demonstrated rapid generalization of learning in motion direction discrimination (Liu and Weinshall 2000). This can be understood as an instance of meta-learning, i.e., "learning of learning:" during the training period, subjects do not only learn to discriminate the stimuli presented, they also acquire knowledge about the general structure of the task, which may facilitate later transfer of the learning to new stimuli, even though the adaptation to the new stimuli may not be instantaneous. The present results suggest that after listeners had gained substantial experience in the frequency discrimination task, this readaptation to new frequencies occurred within the course of a single session, i.e., less than two hours. 
The present findings, showing large transfer of learning across distant frequencies and comparatively weak frequency-specific effects, are consistent with earlier results by Irvine et al. (2000). These authors also demonstrated a slight frequency-specific learning effect in a frequency discrimination task with high-frequency tones $(5$ and $8 \mathrm{kHz}$ ). Because the posttraining session in that study involved only three runs at one untrained frequency in a single ear, it remains possible that rapid generalization of learning between the trained and the untrained frequency would have been observed, in that study too, if the posttraining session had included more runs. In the present study, we found that after only one run at each of three untrained frequencies in two ears (a total of six runs at untrained frequencies), FDTs at untrained frequencies were significantly less improved than those at trained frequencies. However, what we also found is that only a few additional runs were sufficient for the improvement at untrained frequencies to become statistically indistinguishable from that at the trained frequency. Considering that the trained-frequency advantage evidenced in Irvine et al.'s (2000) results was very weak, and in fact just about significant $(p=0.049)$, it seems likely that such a fragile advantage could have rapidly vanished if listeners had been given a little more opportunity to practice at the untrained frequency during the posttraining session. Recent results by Stickney et al. (personal communication) also indicate a large amount of learning transfer between widely separated frequencies (400 and $6000 \mathrm{~Hz}$ ) and comparatively weak frequency-specific effects. Ari-Even Roth et al. (2003) also obtained results which they interpreted as indicating complete generalization of learning across frequency $(1,1.1$, and $2 \mathrm{kHz})$. Unfortunately, this interpretation is not warranted because, in that study, the pre- and posttraining thresholds were always measured first at the frequency that was used as standard during the training.

The present results contrast markedly with the earlier results by Demany and Semal (2002), which suggest that frequency discrimination learning takes a long time to generalize to novel frequencies after training at a single frequency. Indeed, Demany and Semal (2002) showed that following initial training at $3000 \mathrm{~Hz}$, FDTs at two new frequencies (1200 and $6500 \mathrm{~Hz}$ ) continued to decrease for several hours as listeners were now trained at these frequencieswhile at the same time, FDTs at $3000 \mathrm{~Hz}$, which had already reached an asymptotic level earlier, showed no significant further improvement. However, as suggested above, Demany and Semal did not check whether, under the stimulus conditions used in their study, FDTs did not stop to improve earlier at 3000 $\mathrm{Hz}$ than they would have at 1200 and $6500 \mathrm{~Hz}$ if these frequencies had been used as initial training frequencies; therefore, it is difficult to ascertain that the results of that study did not overestimate the frequency specificity of frequency discrimination learning. On the other hand, one might argue that the present study may have underestimated the frequency-specificity of learning because the listeners received nonspecific training prior to frequency-specific training. Results in the visual modality suggest that even very brief exposure, prior to this training, to stimuli that are not used during the training may promote transfer of learning (Ahissar and Hochstein 1997). Admittedly, the frequency-unspecific pretraining session in the present study was rather long, as it involved several hundreds of trials at each of the four frequencies tested. Nonetheless, at all test frequencies except $1500 \mathrm{~Hz}$, substantial improvements in thresholds were observed during the course of the frequency-specific training sessions that followed. It is traditionally assumed that long-term improvements in thresholds reflect primarily sensory learning, which, if subtended by local changes in tonotopically organized maps in the auditory cortex, should not generalize to other frequencies.

Other results that have been interpreted as evidence that frequency discrimination learning is frequency-specific are cited in Wright and Fitzgerald (2005). In that study, listeners were trained in frequency discrimination with pure tones around $1 \mathrm{kHz}$. Before and after training, they were also tested at $4 \mathrm{kHz}$. The results showed a smaller improvement at the untrained frequency $(4 \mathrm{kHz})$ than at the trained frequency $(1 \mathrm{kHz})$. However, the interpretation of those results is complicated by the fact that the improvement in the trained condition was rather smalla factor of about 1.3 on average, compared to a factor of 2 or more in most other studies on pure-tone frequency discrimination learning considered here, including the present one-and in fact, turned out not to be significantly different from that measured in control listeners who only took part in the pre- and posttraining sessions, with no frequency-specific training in between. Considering that, in the present study, the improvements in FDTs observed during the course of the frequency-specific training period were rather large (in some case, FDTs improved by more than a factor of two over that period), it seems unlikely that similar improvements would have been obtained if listeners had only taken part in the preand posttraining sessions.

\section{Transfer of learning across ears}

While some slight but significant frequency specificity of frequency discrimination learning was found, no 
significant effect of ear was observed. The FDTs measured in the left and right ears were found to be statistically similar, and which ear was used during the training had no significant influence on the results. This outcome is consistent with the earlier results of Demany and Semal (2002) and Ari-Even Roth et al. (2004), which also failed to show a significant influence of training ear in frequency discrimination learning. On the other hand, Delhommeau et al. (2002) did find a slight but significant difference in FDTs between the right and left ears following monaural training in frequency discrimination. Furthermore, we have additional data (to be submitted) from a study that was devoted to examining this question in more detail, which also suggest a slight (and under some conditions, statistically significant) advantage for the trained ear. One possible interpretation of these different outcomes is that frequency discrimination learning is perhaps weakly ear specific, but the ear specificity is so slight that it does not reach the threshold of statistical significance in all studies. Alternatively, or complementarily, interindividual differences may exist, so that even if two studies used exactly the same experiment design and had exactly the same statistical power, they might nevertheless produce different outcomes in that respect. Thus, the only conclusion that can safely be drawn based on the currently available data is that frequency discrimination learning depends at best weakly on which ear was used during the training; most or all of the learning generalizes readily across the two ears.

\section{The mechanisms of frequency discrimination learning in humans}

While differing in the details of their design, results, and interpretations, the present and previously published studies on frequency discrimination learning all agree on at least one point: they show large frequency- and ear-unspecific improvements and either no, or comparatively much smaller, frequency- or earspecific effects (Ari-Even Roth et al. 2003; Demany 1985; Demany and Semal 2002; Irvine et al. 2000). For instance, in Irvine et al.'s (2000) study, despite an overall highly significant improvement in FDTs between the pre- and posttraining sessions $(p=0.00001)$, the frequency-specific component of learning was barely statistically significant $(p=0.049)$. Even in Demany and Semal's (2002) study, which suggests perhaps the largest frequency-specific effects so far, FDTs at untrained frequencies $(1200$ and $6500 \mathrm{~Hz})$ were already substantially reduced (by a factor of 3 to 4) just after protracted training at $3000 \mathrm{~Hz}-$ although at that stage of the experiment, the listeners had received little exposure to these untrained frequencies. In the present study, FDTs at the trained frequency improved by a factor of about 2 between the pre- and posttraining sessions, whereas the largest difference between FDTs at trained and untrained frequencies on the post training session was only around $20 \%$.

These findings in humans contrast markedly with those of Recanzone et al. (1993) in monkeys. In that study, thresholds at untrained frequencies were substantially larger than thresholds at the trained frequency. In trying to explain this discrepancy between human and monkey data, several points are worth considering. First, the incentives for learning in the human and animal studies were different: while in monkeys correct responses were rewarded with food, humans simply received visual feedback. Thus, to the extent that motivation influences learning, different learning effects are to be expected in humans and animals. In addition, humans and laboratory animals evolve in very different environments; stimulations in laboratory environments are typically rarer and much less diverse than in natural environments. As a result, stimuli presented during the experiments may have a stronger impact and longer-lasting effects on laboratory animals than on humans. Alternatively, the mechanisms of auditory frequency discrimination learning may be different in humans and monkeys. Some evidence for interspecies difference in training-induced plasticity is provided by a recent report by Brown et al. (2004), which indicates that, in cats, frequency discrimination learning is not paralleled by enlarged representations of the training frequencies in the animal's A1, contrary to Recanzone et al.'s (1993) findings in monkeys. The comparatively large frequency unspecificity of frequency discrimination learning in humans is not compatible with local modifications in tonotopic maps being the main mechanism behind practice-related improvements in FDTs. Instead, it appears that, in humans, pure-tone frequency discrimination learning is mediated mainly-although not entirely-by mechanisms that do not depend critically on the frequency region in which the two stimuli that the listener must compare are presented.

\section{Different mechanisms at low and high frequencies?}

As mentioned in the Introduction, it has been suggested that frequency discrimination is mediated by temporal (phase locking) mechanisms at low frequencies $(<4-5 \mathrm{kHz})$, and by tonotopic or placebased mechanisms at higher frequencies (Moore 1973; Rose et al. 1968). The present results provide no evidence for a dissociation between $6 \mathrm{kHz}$ and lower frequencies. Frequency discrimination learning 
was not found to be any weaker or stronger at $6 \mathrm{kHz}$ than at lower frequencies, nor was it found to generalize less or more between $6 \mathrm{kHz}$ and lower frequencies than among the latter. A possible reason for this negative outcome is that, at the stage of auditoryinformation processing where frequency discrimination learning takes place, low and high frequencies are represented using a common neural code.

\section{ACKNOWLEDGMENTS}

The work was supported in part by a research grant from the French Ministry of Research and Education (Action Cognitique 2000, Art et Cognition, Project \#A62) to C. Micheyl (then at UMR CNRS 5020, Lyon, France), CNRS (France), and NIH/NIDCD grant R01 DC05216 to A.J. Oxenham (MIT, RLE, Cambridge, MA). We thank Laurent Demany, Armin Kohlrausch, and one anonymous reviewer for helpful comments on an earlier version of this manuscript.

\section{REFERENCES}

AhISSAR M. Perceptual training: a tool for both modifying the brain and exploring it. Proc. Natl. Acad. Sci. USA 98:11842-11843, 2001.

Ahissar M, Hochstein S. Learning pop-out detection: specificities to stimulus characteristics. Vis. Res. 36:3487-3500, 1996.

Ahissar M, Hochstein S. Task difficulty and the specificity of perceptual learning. Nature 387:401-406, 1997.

Ari-Even RD, Amir O, Alaluf L, Buchsenspanner S, Kishon-Rabin L. The effect of training on frequency discrimination: generalization to untrained frequencies and to the untrained ear. J. Basic Clin. Physiol. Pharmacol. 14:137-150, 2003.

Ari-Even RD, Avrahami T, Sabo Y, Kishon-Rabin L. Frequency discrimination training: is there ear symmetry? J. Basic Clin. Physiol. Pharmacol. 15:15-27, 2004.

Ball K, Sekuler R. A specific and enduring improvement in visual motion discrimination. Science 218:697-698, 1982.

Brown M, Irvine DR, PARK VN. Perceptual learning on an auditory frequency discrimination task by cats: association with changes in primary auditory cortex. Cereb. Cortex 14:952-965, 2004.

Campbell RA, Small AM. Effect of practice and feedback on frequency discrimination. J. Acoust. Soc. Am. 35:1511-1514, 1963.

Delhommeau K, Micheyl C, Jouvent R, Collet L. Transfer of learning across durations and ears in auditory frequency discrimination. Percept. Psychophys. 64:426-436, 2002.

Demany L. Perceptual learning in frequency discrimination. J. Acoust. Soc. Am. 78:1118-1120, 1985.
Demany L, Semal C. Learning to perceive pitch differences. J. Acoust. Soc. Am. 111:1377-1388, 2002.

FioRENTINI A, BERARdi N. Perceptual learning specific for orientation and spatial frequency. Nature 287:43-44, 1980.

FioRENTINI A, BERARDi N. Learning in grating waveform discrimination: specificity for orientation and spatial frequency. Vis. Res. 21:1149-1158, 1981.

GiLBERT CD. Learning. Neuronal dynamics and perceptual learning. Curr. Biol. 4:627-629, 1994.

GiLBert CD. Learning and receptive field plasticity. Proc. Natl. Acad. Sci. USA. 93:10546-10547, 1996.

Grimault N, Micheyl C, Carlyon RP, Collet L. Evidence for two pitch encoding mechanisms using a selective auditory training paradigm. Percept. Psychophys. 64((2)):189-197, 2002.

Grimault N, Micheyl C, Carlyon RP, Bacon SP, Collet L. Learning in discrimination of frequency or modulation rate: generalization to fundamental frequency discrimination. Hear. Res. 184(1-2):41-50, 2003.

Hawkey DJ, Amitay S, Moore DR. Early and rapid perceptual learning. Nat. Neurosci. (2004). Advanced online publication.

Irvine DR, Martin RL, KuimkeIT E, SMith R. Specificity of perceptual learning in a frequency discrimination task. J. Acoust. Soc. Am. 108:2964-2968, 2000.

KARNi A, Bertini G. Learning perceptual skills: behavioral probes into adult cortical plasticity. Curr. Opin. Neurobiol. 7:530-535, 1997.

KARNi A, SAGI D. Where practice makes perfect in texture discrimination: evidence for primary visual cortex plasticity. Proc. Natl. Acad. Sci. USA 88:4966-4970, 1991.

KARNi A, SAGi D. The time course of learning a visual skill. Nature 365:250-252, 1993.

Liu Z, Weinshall D. Mechanisms of generalization in perceptual learning. Vis. Res. 40:97-109, 2000.

Moore BC. Frequency difference limens for short-duration tones. J. Acoust. Soc. Am. 54:610-619, 1973.

Recanzone GH, Schreiner CE, Merzenich MM. Plasticity in the frequency representation of primary auditory cortex following discrimination training in adult owl monkeys. J. Neurosci. 13:87-103, 1993.

Robinson K, Summerfield AQ. Adult auditory learning and training. Ear Hear. 17:51S-65S, 1996.

Rose Je, Brugge JF, Anderson DJ, Hind JE. Patterns of activity in single auditory nerve fibres of the squirrel monkey. In: de Reuck AVS, Knight J (eds) Hearing Mechanisms in Vertebrates. Churchill, London, 1968.

Schoups AA, Orban GA. Interocular transfer in perceptual learning of a pop-out discrimination task. Proc. Natl. Acad. Sci. USA 93:7358-7362, 1996.

Wright BA, FitzGerald MB. Learning and generalization of five auditory discrimination tasks as assessed by threshold changes. In: Pressnitzer D, de Cheveigne A, McAdams S, Collet L (eds) Auditory Signal Processing: Physiology, Psychoacoustics, and Models. Springer, New York, 2005.

Wright BA, Buonomano DV, Mahncke HW, Merzenich MM. Learning and generalization of auditory temporal-interval discrimination in humans. J. Neurosci. 17:3956-3963, 1997. 